\title{
Mid-life extra-haematopoetic manifestations of Diamond-Blackfan anaemia
}

\author{
Christopher Muir1, Anthony Dodds² and Katherine Samaras1,3 \\ Departments of 'Endocrinology, ${ }^{2 H a e m a t o l o g y}$ and Bone Marrow Transplantation, St Vincent's Hospital,
} Sydney, Australia, and ${ }^{3}$ Garvan Institute of Medical Research, Sydney, Australia

Correspondence should be addressed to K Samaras

Email

k.samaras@garvan.org.au

\section{Summary}

Diamond-Blackfan anaemia (DBA) is a rare cause of bone marrow failure. The incidence of malignancy and endocrine complications are increased in DBA, relative to other inherited bone marrow failure syndromes. We describe an adult woman with DBA who developed osteoporosis and avascular necrosis (AVN) of both distal femora. Such endocrine complications are not uncommon in DBA, but under-appreciated, especially in adulthood. Further, rectal adenocarcinoma was diagnosed at age 32 years, requiring hemi-colectomy and adjuvant chemotherapy. Elevated cancer risk may warrant disease-specific screening guidelines. Genetic predictors of extra-haematopoetic complications in DBA are yet to be established.

\section{Learning points:}

- Endocrine complications are common in DBA.

- Clinical vigilance is required in managing bone health of DBA patients treated with glucocorticoids.

- There is currently no reliable way to predict which patients will develop complications of therapy or premature malignancy related to DBA.

- Complaints of bone or joint pain should prompt screening with targeted magnetic resonance imaging. Osteoporosis screening should be performed routinely.

\section{Background}

Diamond-Blackfan anaemia (DBA) is a rare disease resulting in pure red cell aplasia (1). DBA most commonly arises secondary to haplo-insufficiency of the RPS19 gene, resulting in a dysfunctional ribosomal protein (2). After identification of RPS19 in 1999, further genetic mutations have been described (3). Approximately half of all cases are inherited in an autosomal dominant fashion, with the remainder being sporadic or via other inheritance patterns (4).

DBA is typically glucocorticoid responsive, and chronic therapy remains the treatment mainstay (5). The mechanism of the therapeutic effect is unknown, but is distinct from immunosuppressant effects. Responders cannot be reliably identified phenotypically- or genotypically. Prednisone doses are tapered to the lowest required to maintain acceptable haemoglobin levels, with great inter-individual variations in the dose required. Duration of therapy is also variable and poorly predictable, with some patients developing glucocorticoid refractoriness and others eventual remission $(5,6)$. Endocrine complications may be present in over half of affected patients, related to chronic glucocorticoid use or recurrent transfusion and secondary iron overload (7). Furthermore, congenital anomalies and predisposition to malignancy commonly form part of the clinical spectrum $(8,9)$. 
Table 1 Bone mineral density results (2002-2016) of the lumbar spine, right femoral neck and right total proximal femur with concurrent prescribed osteoporosis therapy.

\begin{tabular}{l}
\hline Site \\
\hline Lumbar spine (L2-L4) \\
BMD \\
T-score \\
Femoral neck \\
BMD \\
T-score \\
Total hip \\
BMD \\
T-score
\end{tabular}

\begin{tabular}{c}
\hline Mar '02 \\
\hline 1.07 \\
-1.1 \\
0.82 \\
-1.3
\end{tabular}

\begin{tabular}{c}
\hline Oct ‘04 \\
\hline 0.98 \\
-1.8 \\
0.79 \\
-1.6
\end{tabular}

\begin{tabular}{c}
\hline Apr '06 \\
\hline 1.03 \\
-1.5
\end{tabular}

\begin{tabular}{c}
\hline May '07 \\
\hline 1.06 \\
-1.3
\end{tabular}

\begin{tabular}{c}
\hline Dec '08 \\
\hline 1.08 \\
-1.1
\end{tabular}

\begin{tabular}{c}
\hline Feb '13 \\
\hline 1.09 \\
-1.1
\end{tabular}

\begin{tabular}{cll}
\hline Dec '14 & & Feb '16 \\
\cline { 1 - 1 } 1.07 & & 1.10
\end{tabular}

T-score

Alendronate*

Cholecalciferol

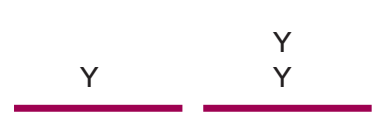

$\begin{array}{r}0.72 \\ -2.5 \\ Y \\ Y \\ \hline\end{array}$

*Alendronate was given for 18 months.

We report the case of a 45 -year-old woman with DBA who developed osteoporosis and avascular necrosis (AVN), as well as invasive rectal cancer.

\section{Case presentation}

The diagnosis of DBA was made via bone marrow biopsy at 6 months of age after presenting with failure to thrive. The pathogenic mutation has not been established. Variable dose glucocorticoids were used in a cyclical pattern since diagnosis: initially $5 \mathrm{mg}$ for 2 days, 14 days off (age 12-20 years); $5 \mathrm{mg}$ for 3 days, 10 days off (age 21-35 years); $15 \mathrm{mg}$ for 3 days, 10 days off (age 35-44 Years), with a short period of haemoglobin instability at age 20 years necessitating prednisone doses up to $25 \mathrm{mg}$ daily for a period of 12 months. Since age 44 years, cautious reductions have been undertaken to $12.5 / 14.5 / 14.5 \mathrm{mg}$ on three consecutive days with 10 days off, $\sim 0.05 \mathrm{mg} / \mathrm{kg} /$ day), without anaemia relapse. Further dose reduction trials are to be undertaken under close medical supervision.

Endocrine assessment occurred at age 30 years, where multiple endocrine complications related to chronic glucocorticoid use were evident, including central adiposity, osteoporosis and multi-centric avascular necrosis. Bone mineral density testing demonstrated osteopenia of the femoral neck and lumbar spine. There was no history of fracture. Apart from glucocorticoids, secondary causes of osteoporosis were excluded. Calcium and vitamin D supplementation were commenced. At age 33 years, a decline in bone mineral density prompted initiation of oral bisphosphonate therapy with alendronate $70 \mathrm{mg}$ weekly. Serial bone mineral density measurements improved, and alendronate was ceased after 18 months of therapy (Table 1 ).
Atraumatic bilateral knee pain developed at age 39 years. Dedicated radiographs were unremarkable. Magnetic resonance imaging showed a $2.5 \times 1.2 \mathrm{~cm}$ area of AVN in the left lateral femoral condyle (Fig. 1), with additional foci in the right lateral and medial femoral condyles. Bisphosphonate therapy was reconsidered, but not recommenced, as bone turnover markers remained suppressed. Radionuclide bone scan did not show any evidence of active bone devascularisation, and follow-up MRI demonstrated stable bone architecture.

At age 33 years, a tubular adenocarcinoma of the rectum was detected, managed with low anterior resection and cautious adjuvant 5-fluorouracil chemotherapy. Anatomical pathology showed evidence of intramural and extramural spread with perineural invasion. Immunoperoxidase staining was positive for MLH1, MSH2 and MSH6, indicating a low likelihood of microsatellite instability. Surveillance colonoscopies have been clear of disease since, and tumour markers remain undetectable. There is no family history of colon cancer.

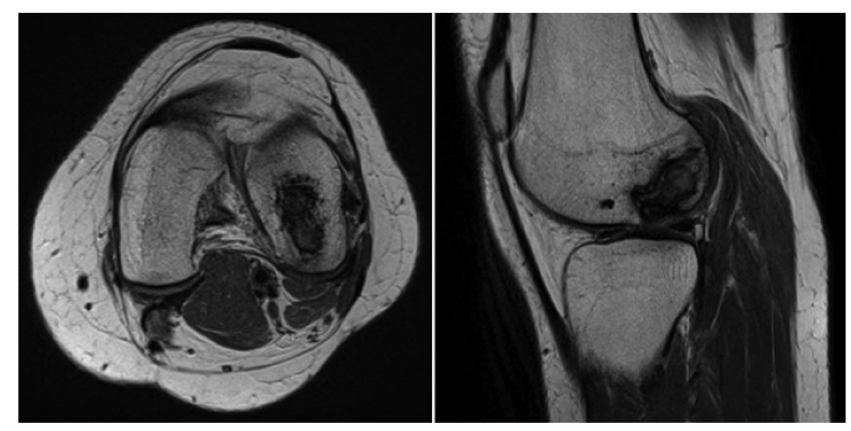

Figure 1

Magnetic resonance imaging of the left knee, with associated area of avascular necrosis affecting the left lateral femoral condyle. 


\section{Discussion}

DBA is an established ribosomopathy resulting in hypoplastic anaemia and bone marrow failure. Treatment usually involves long-term glucocorticoid therapy and in a subset of patients, recurrent transfusions are required. Treatment-related adverse effects are increasingly recognised among this patient population due to their survival into mid- and later-life.

Data are emerging on the endocrine complications in later life: American DBA registry data reported that 53\% $(n=57)$ had one or more endocrine complication (7), most commonly adrenal insufficiency (32\%), hypogonadism (29\%) and hypothyroidism (14\%). Vitamin D deficiency was reported in 57\%. Few data have been published on bone health in DBA, despite the strong association between chronic glucocorticoid use and bone loss (10). For example, the previously mentioned study evaluated bone density in only a small subset (15\%) (7), and pathological fractures were separately reported in $22 \%$ (8). Similarly, a Japanese study of 57 DBA patients treated with prednisone and/or stem cell transplantation reported glucocorticoid-related complications in $22 \%$ (11). The majority of reported data are derived from a paediatric population, with limited data specific to adult patients and leaving the true prevalence and burden of glucocorticoid-induced bone loss relatively unknown. Further, to our knowledge, there are no data published on responses to standard osteoporosis treatments in DBA. As such, documentation of cases demonstrating beneficial effect of short-term bisphosphonate in mid-adulthood and complications such as multi-centric AVN contribute to the picture of bone health in survivors.

$\mathrm{AVN}$ is an under-recognised complication of DBA, despite its strong association with glucocorticoid use (10). The overall incidence rate of glucocorticoid-associated AVN has been estimated to be up to 25\% (12). At present, there are only four other reported cases of AVN in DBA: a 17-year-old man with bilateral osteonecrosis of the femoral heads who progressed to bilateral total hip replacement (13), atraumatic AVN of the patella in an adult woman (14) and two additional cases reported incidentally as part of larger studies examining other aspects of DBA $(7,15)$.

DBA is associated with an increased incidence of cancer, which occurs at a lower median age compared to the general population (16). Until recently, information regarding cancer risk in DBA was limited to case reports and small case series. The majority of reported cases were haematological, and it was considered that solid tumour malignancies were relatively less common. However, recent prospective registry data in 608 patients reported 17 malignancies over 9458 person-years follow-up (16). Documented solid organ cancers included colonic adenocarcinoma (3 cases), sarcoma (3 cases), breast cancer ( 2 cases), squamous cell carcinoma (2 cases) and single cases of melanoma, uterine, cervical and testicular cancer. Haematological malignancies included myelodysplasia (4 cases), acute myeloid leukaemia (3 cases) and non-Hodgkin lymphoma (1 case). Taken together, DBA is associated with a 5.4-fold increase in cancer risk compared to the general population, with the odds ratio being the highest for MDS (237.0) and colonic adenocarcinoma (36.2) (16).

Reduced cancer-related survival has also been described. Neutropaenic sepsis and marrow suppression result in chemotherapy delay, contributing to the poor outcomes seen in comparison to a DBA-unaffected population (16). As in our patient, the three reported cases of colonic adenocarcinoma in the DBA registry cohort occurred at relatively young ages (34, 43 and 49 years) (16). In one case (age 34 years), there was a known RPS19 gene mutation, whereas the clinical genetics were unknown for the other 2 affected individuals (16). To date, no clear association between genetic defect and future cancer risk in DBA has been established, with over $50 \%$ of DBA cases being attributable to mutations in 17 genes coding various ribosomal proteins (17). It is unclear whether additional cancer screening (faecal occult blood testing, colonoscopy, breast examination/ultrasound, etc.) should be performed at an earlier age in people with DBA compared with the general community and whether it might improve outcomes.

Endocrine complications are common in DBA, with chronic glucocorticoid therapy being a strong aetiologic factor. Osteoporosis, fragility fractures and atraumatic AVN are under-recognised and underreported complications, especially in adult survivors with DBA. To our knowledge, this is the first reported case of AVN affecting the distal femur in a patient with DBA and one of few reports documenting osteoporosis, treatment and monitoring with recurrent bone mineral density measurements. As glucocorticoids are the mainstay of DBA treatment, case reports such as this highlight the need for clinical vigilance in managing bone health in DBA patients. Complaints of bone and/or joint pain should prompt screening with targeted magnetic resonance imaging. It remains unclear whether DBA predisposes to bone loss in its own right, but irrespective, osteoporosis screening with bone mineral densitometry should be undertaken and repeated at regular intervals to ensure early identification and treatment of 
glucocorticoid-induced bone loss. Additionally, other secondary causes of osteoporosis (i.e. hypogonadism, vitamin D deficiency) should be screened and treated when present. DBA conveys a predisposition to solid organ and haematological malignancy, often at ages much younger than those seen in the general population. Current population-based recommendations for cancer screening are unlikely to adequately serve this patient population, and disease-specific guidelines may be required. There is currently no reliable way to predict which patients will develop cancer. Further research into clinical and genetic predictors of malignancy in DBA is needed.

\section{Patient's perspective}

The patient wishes to acknowledge the pioneering work of Prof Darcy O'Gorman Hughes (1930-2000), whose clinical acumen in accuracy of diagnosis in the absence of today's technologies and care through childhood impacted immensely and positively on her health and flourishing in everyday life.

\section{Declaration of interest}

The authors declare that there is no conflict of interest that could be perceived as prejudicing the impartiality of the research reported.

\section{Funding}

This research did not receive any specific grant from any funding agency in the public, commercial or not-for profit sector.

\section{Patient consent}

Written consent has been obtained from the patient prior to submission of this report.

\section{Author contribution statement}

Christopher Muir prepared the original manuscript and was involved in all subsequent revisions. Anthony Dodds was the treating haematologist and was involved in manuscript revisions. Katherine Samaras was the treating endocrinologist and was involved in manuscript revisions.

\section{References}

1 Diamond L \& Blackfan K 1938 Hypoplastic anemia. American Journal of Diseases of Children $\mathbf{5 6}$ 464-467.

2 Draptchinskaia N, Gustavsson P, Andersson B, Pettersson M, Willig T, Dianzani I, Ball S, Tchernia G, Klar J, Matsson H, et al.
1999 The gene encoding ribosomal protein S19 is mutated in Diamond-Blackfan anaemia. Nature Genetics 21 169-175. (doi:10.1038/5951)

3 Gazda H, Sheen M, Darras N, Schneider H, Sieff C, Ball S, Niewiadomska E, Newburger PE, Atsidaftos E, Vlachos A, et al. 2007 Mutations of the genes for ribosomal proteins L5 and L11 are a common cause of Diamond-Blackfan anemia. Blood 110421.

4 Orfali K, Ohene-Abuakwa Y \& Ball S 2004 Diamond-Blackfan anaemia in the UK: clinical and genetic heterogeneity. British Journal of Haematology 125 243-252. (doi:10.1111/j.1365-2141.2004.04890.x)

5 Vlachos A, Ball S, Dahl N, Alter B, Sheth S, Ramenghi U, Meerpohl J, Karlsson S, Liu JM, Leblanc T, et al. 2008 Diagnosing and treating Diamond-Blackfan anaemia: results of an international clinical consensus conference. British Journal of Haematology 142 859-876. (doi:10.1111/j.1365-2141.2008.07269.x)

6 Vlachos A \& Muir E 2010 How I treat Diamond-Blackfan anemia. Blood 116 3715-3723. (doi:10.1182/blood-2010-02-251090)

7 Lahoti A, Harris Y, Speiser P, Atsidaftos E, Lipton J \& Vlachos A 2016 Endocrine dysfunction in Diamond-Blackfan anemia: a report from the DBA registry. Pediatric Blood and Cancer 62 306-312. (doi:10.1002/ pbc. 25780 )

8 Lipton J, Atsidaftos E, Zyskind I \& Vlachos A 2006 Improving clinical care and elucidating the pathophysiology of Diamond-Blackfan anemia: an update from the Diamond-Blackfan anemia registry. Pediatric Blood and Cancer 46 558-564. (doi:10.1002/pbc.20642)

9 Ball SE, McGuckin CP, Jenkins G \& Gordon-Smith E 1996 Diamond-Blackfan anaemia in the U.K.: analysis of 80 cases from a 20 year birth cohort. British Journal of Haematology 94 645-653. (doi:10.1046/j.1365-2141.1996.d01-1839.x)

10 Weinstein R 2011 Glucocorticoid-induced bone disease. New England Journal of Medicine 365 62-70. (doi:10.1056/NEJMcp1012926)

11 Ogha S, Mugishima H, Ohara A, Kojima S, Fujisawa K, Yagi K, Higashigawa M, Tsukimoto I \& Aplastic Anemia Committee Japanese Society of Pediatric Hematology 2004 Diamond-Blackfan anemia in Japan: clinical outcomes of prednisolone therapy and hematopoetic stem cell transplantation. International Journal of Hematology 79 22-30. (doi:10.1007/BF02983529)

12 Mankin H 1992 Nontraumatic necrosis of bone (osteonecrosis). New England Journal of Medicine 326 1473-1479. (doi:10.1056/ NEJM199205283262206)

13 Velez K, Johnson T \& Wennemer H 2009 Bilateral total hip replacement in a teenager with Diamond Blackfan anemia. Archives of Physical Medicine and Rehabilitation 1 1045-1047. (doi:10.1016/j. pmrj.2009.10.003)

14 Baumgarten KM, Mont MA, Rifai A, Rifai A \& Hungerford D 2001 Atraumatic osteonecrosis of the patella. Clinical Orthopaedics and Related Research 383 191-196. (doi:10.1097/00003086-200102000-00021)

15 Seguin C, Kassis J, Busque L, Bestawros A, Theodoropoulos J, Alonso M \& Harvey E 2008 Non-traumatic necrosis of bone (osteonecrosis) is associated with endothelial cell activation but not thrombophilia. Rheumatology International 47 1151-1155. (doi:10.1093/rheumatology/ken206)

16 Vlachos A, Rosenberg P, Atsidaftos E, Alter B \& Lipton J 2012 The incidence of neoplasia in Diamond-Blackfan anemia: a report from the Diamond-Blackfan Anemia Registry. Blood 119 3815-3819. (doi:10.1182/blood-2011-08-375972)

17 Clinton C \& Gazda HT 1993 Diamond-Blackfan anaemia. In Gene Reviews, Eds RA Pagon, TC Bird, CR Dolan \& K Stephens. Seattle, Washington, USA: University of Washington.

Received in final form 17 February 2017

Accepted 17 February 2017 